\title{
Predictive capability of average Stokes polarimetry for simulation of phase multilevel elements onto LCoS devices
}

\author{
Francisco J. Martínez, ${ }^{1,2}$ Andrés Márquez, ${ }^{1,2, \star}$ Sergi Gallego,,$^{1,2}$ Manuel Ortuño, ${ }^{1,2}$ \\ Jorge Francés, ${ }^{1,2}$ Inmaculada Pascual, ${ }^{2,3}$ and Augusto Beléndez ${ }^{1,2}$ \\ ${ }^{1}$ Dept. de Física, Ingeniería de Sistemas y Teoría de la Señal, Universidad de Alicante, P.O. Box 99, E-03080 Alicante, Spain \\ ${ }^{2}$ Instituto Universitario Física Aplicada a las Ciencias y las Tecnologías, Universidad de Alicante, \\ P.O. Box 99, E-03080 Alicante, Spain \\ ${ }^{3}$ Dept. de Óptica, Farmacología, y Anatomía, Universidad de Alicante, P.O. Box 99, E-03080 Alicante, Spain \\ *Corresponding author: andres.marquez@ua.es
}

Received 1 October 2014; revised 28 December 2014; accepted 14 January 2015;

posted 14 January 2015 (Doc. ID 224110); published 16 February 2015

\begin{abstract}
Parallel-aligned (PA) liquid-crystal on silicon (LCoS) microdisplays are especially appealing in a wide range of spatial light modulation applications since they enable phase-only operation. Recently we proposed a novel polarimetric method, based on Stokes polarimetry, enabling the characterization of their linear retardance and the magnitude of their associated phase fluctuations or flicker, exhibited by many LCoS devices. In this work we apply the calibrated values obtained with this technique to show their capability to predict the performance of spatially varying phase multilevel elements displayed onto the PA-LCoS device. Specifically we address a series of multilevel phase blazed gratings. We analyze both their average diffraction efficiency ("static" analysis) and its associated time fluctuation ("dynamic" analysis). Two different electrical configuration files with different degrees of flicker are applied in order to evaluate the actual influence of flicker on the expected performance of the diffractive optical elements addressed. We obtain a good agreement between simulation and experiment, thus demonstrating the predictive capability of the calibration provided by the average Stokes polarimetric technique. Additionally, it is obtained that for electrical configurations with less than $30^{\circ}$ amplitude for the flicker retardance, they may not influence the performance of the blazed gratings. In general, we demonstrate that the influence of flicker greatly diminishes when the number of quantization levels in the optical element increases. (C) 2015 Optical Society of America

OCIS codes: (050.1970) Diffractive optics; (120.2040) Displays; (120.5410) Polarimetry; (230.3720)

Liquid-crystal devices; (230.6120) Spatial light modulators; (230.2090) Electro-optical devices.

http://dx.doi.org/10.1364/AO.54.001379
\end{abstract}

\section{Introduction}

Phase-only modulation is necessary for many spatial light modulation applications, such as diffractive optics [1], optical storage [2,3], optical metrology [4], reconfigurable interconnects $[\underline{5}, 6]$, wavefront sensing of structured light beams [7] , holographic optical traps [8], or quantum optical computing [9]. Among

1559-128X/15/061379-08 $\$ 15.00 / 0$

(C) 2015 Optical Society of America the available spatial light modulator (SLM) technologies [10], parallel-aligned (PA) liquid-crystal on silicon $(\overline{\mathrm{LCoS}})$ microdisplays are especially appealing since they enable phase-only operation without coupled amplitude modulation. They are electrooptic devices which can be assimilated to linear variable retarders. Therefore, their performance is characterized by their linear retardance as a function of the applied voltage. However, it has been found by numerous researchers [11-18] that in general, LCoS (both parallel-aligned and twisted nematic) produces 
phase flicker and/or depolarization. This is true for the ones with a digital backplane [13] due to the pulsed nature of the voltage signal addressed $[12,13]$, but it may also be true in analogically addressed due to charge leakage in the pixel between consecutive refreshing frames.

Recently some methods have been proposed to characterize not only the linear retardance but also the magnitude of phase flicker in the optical signal [19-21] specifically valid for parallel aligned liquid-crystal devices (LCDs). In particular, we have demonstrated the predictive capability of a timeaveraged Stokes polarimetric technique [21], and we have analyzed its robustness and its applicability to characterize PA-LCoS devices in a wide variety of working conditions [22].

In Refs. [21,22] the PA-LCoS was addressed by a uniform voltage image; however, in spatial light modulation applications spatially varying signals are actually applied. Then it would be interesting both to learn about the influence of the phase flicker and also to check the predictive capability of the calibrated values we have obtained when used in more complex situations. SLMs are widely used in diffractive optics, and one of the most extensively used diffractive optical elements (DOEs) is the blazed grating. Due to its multilevel structure and to its well-defined spatial frequency it is a very proper increase in complexity with respect to uniform voltage images. The effects of flicker in blazed gratings were analyzed in [23]. However, estimated flicker amplitude values used were not that precise as the ones enabled by average Stokes polarimetry. Additionally, only a very specific long-period blazed grating was considered [23]. Focus was also given to the application of the minimum Euclidean approach to phaseonly SLMs exhibiting flicker and with a phase dynamic range smaller than $2 \pi$. In this paper the phase dynamic range is $2 \pi$ and we consider a much favorable and precisely measured flicker amplitude scenario. This enables us to quantitatively analyze the validity of the calibration previously performed with the Stokes average method and to use these more exact values to show the actual influence of flicker in the behavior of the PA-LCoS device in a spatially varying spatial light modulation application. To this goal we will address $2 \pi$ phase depth blazed gratings, with different spatial periodicities and with different numbers of quantization levels. Specifically we will apply one pixel per level, so that an $N$-level blazed grating has an $N$-pixel period. Both the static performance and the dynamic variation of the diffraction efficiencies will be analyzed, and a comparison between experiment and simulation will provide us with some profitable conclusions.

\section{Retardance and Flicker Calibration}

The phase flicker exhibited by LCoS devices within the frame period has been found to be properly approximated by a triangular time-dependent profile as shown in Refs. $[\underline{16}, \underline{21}]$. If we consider the averaged value for a measurement integration time equal or larger than the addressing frame period what we get is that the state of polarization (SOP) for the light beam reflected by the LCoS may not be fully polarized. This leads us to use the Mueller-Stokes formalism, which enables us to deal both with polarized and unpolarized light [24] to describe the action of PALCoS displays on the light beam. Applying this formalism and taking into account the triangular time-dependent profile for the linear retardance, we obtained Matrix (1) for a linear variable retarder with flicker

$$
\left\langle M_{R}(\bar{\Gamma}, a)\right\rangle=\left(\begin{array}{cccc}
1 & 0 & 0 & 0 \\
0 & 1 & 0 & 0 \\
0 & 0 & (\sin a / a) \cos \bar{\Gamma} & (\sin a / a) \sin \bar{\Gamma} \\
0 & 0 & -(\sin a / a) \sin \bar{\Gamma} & (\sin a / a) \cos \bar{\Gamma}
\end{array}\right),
$$

where $\bar{\Gamma}$ is the average retardance within the frame period, and $a$ its fluctuation amplitude (defined as half the maximum-to-minimum value for the fluctuation). To calibrate the values for these two parameters in the model we measured the averaged Stokes vector parameters for an input light beam linearly polarized light at $+45^{\circ}$ with respect to the $x$-axis (i.e., $S_{0}=1, S_{1}=0, S_{2}=1$, and $S_{3}=0$ ). The analytical expressions for the reflected averaged Stokes vector $\left\langle S_{\text {out }}\right\rangle$ and for the degree of polarization (DoP) are very simple in this case

$$
\begin{gathered}
\left\langle S_{\text {out }}\right\rangle=\left(\begin{array}{c}
1 \\
0 \\
-(\sin a / a) \cos \bar{\Gamma} \\
(\sin a / a) \sin \bar{\Gamma}
\end{array}\right), \\
\operatorname{DoP}=(\sin a / a) .
\end{gathered}
$$

Eventually, the calibration can be easily accomplished using Eq. (3) to obtain the fluctuation amplitude $a$ and the ratio between the third and fourth Stokes vector components, i.e., $-\left\langle S_{3}\right\rangle /\left\langle S_{2}\right\rangle=\operatorname{tg}(\bar{\Gamma})$, to obtain $\bar{\Gamma}[21]$.

In the present work we consider a commercial PA-LCoS display, model PLUTO distributed by the company HOLOEYE. It is filled with a nematic liquid crystal, with 1920 pixels $\times 1080$ pixels and 0.7 in. diagonal, $8 \mu \mathrm{m}$ pixel pitch, $83 \%$ fill factor, and digitally addressed. By means of a RS-232 interface and its correspondingly provided software, we can configure the modulator for different applications and wavelengths. Besides, different pulse width modulation (PWM) addressing schemes (digital addressing sequences) can be generated by the driver electronics $[12,25]$. We consider two electrical sequences exhibiting a clearly different scale of fluctuations, whose configuration files are provided with the software. They correspond to the configurations labeled as "18-6 633 2pi linear" and "5-5 633 2pi 


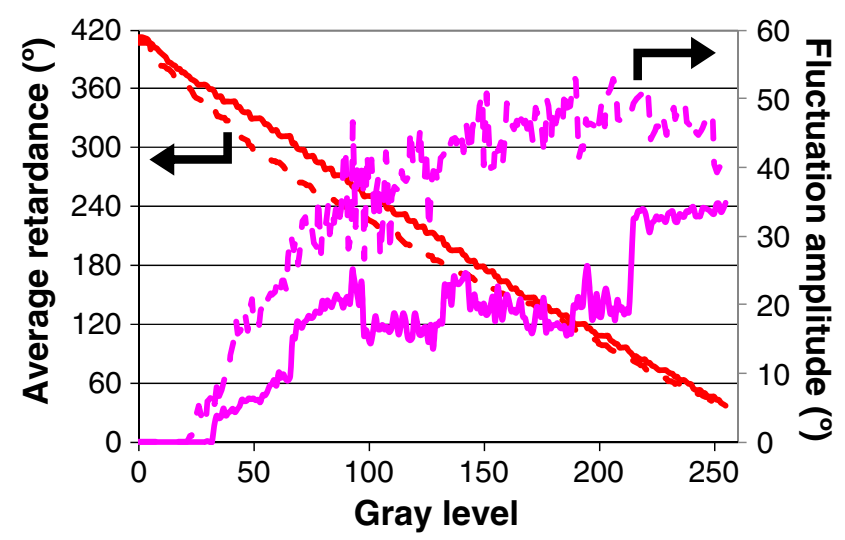

Fig. 1. Calculated values for average retardance and fluctuation amplitude for $\lambda=633 \mathrm{~nm}$, and for sequences "18-6 633 2pi linear" (dashed) and "5-5 633 2pi linear" (continuous) at quasiperpendicular incidence at $3^{\circ}$.

linear." The actual number of quantization values available with these configuration files is respectively 256 and 92 . If necessary, in the case of the 5-5 sequence it is possible to increase the number of available quantization levels up to 192 as shown in [25].

The working geometry considered in this paper corresponds to quasi-perpendicular incidence [22] (actual angle of incidence is $3^{\circ}$ ). We note that the $\overline{\text { di- }}$ rector axis (extraordinary axis) in nematic-based LCoS generally corresponds to the slow axis. In the present LCoS the director axis is along the horizontal. In Fig. 1 we show the calibrated values for the average retardance and its flicker amplitude versus the gray level addressed (voltage), and for the two digital sequences considered. We observe that the retardance range is about $360^{\circ}$ for both sequences with a very good linearity, especially for the sequence "5-5 633 2pi linear." The fluctuation amplitude is clearly smaller for the 5-5 sequence with maximum values slightly higher than $30^{\circ}$. The various jumps encountered in the fluctuation amplitude in both sequences reveal the pulsed nature of the digital signal addressed onto the LCoS: more jumps are seen in the 18-6 sequence. For DoP $>1$, nonphysical values, we consider that fluctuation amplitude is $0^{\circ}$, as can be seen for low gray-level values [21].

\section{Numerical and Experimental Realization: Blazed Gratings}

The calibrated values will be used to simulate the diffraction efficiency for multilevel phase DOEs, in particular blazed gratings. We want to check the influence of the fluctuations. We consider a series of gratings with different number of quantization levels, which due to the pixelated structure of LCoS devices translates into different spatial periodicities: specifically in this work we consider 1 pixel/level. In particular the range considered varies from two to 20 quantization levels, which approaches the limit of a continuous profile. In all cases the gratings have a $360^{\circ}$ phase depth, so that maximum diffraction efficiency in the first order $\left(\eta_{1}\right)$ can be reached, which can be analytically calculated with Eq. (ㅁ) [1]

$$
\eta_{1}=\left(\frac{\sin \left(\frac{\pi}{q}\right)}{\frac{\pi}{q}}\right)^{2}
$$

where $q$ is the number of quantization levels. For example, $\eta_{1}=0.987$ for $q=16$ levels, i.e., basically performs as a continuous profile. On the lower diffraction efficiency limit, $\eta_{1}=0.405$ for $q=2$ levels, which actually corresponds to a binary phase grating with a $180^{\circ}$ phase depth. We also note that for a $360^{\circ}$ phase depth blazed grating the zero-order diffraction efficiency is $\eta_{0}=0$. As an additional remark, in the simulation, even though the calibration curves for the retardance in Fig. 1 show a good linearity, we have applied the appropriate linearization look-up table for each of the two sequences, so that the residual nonlinearity is compensated via software.

We want to note that, as shown in Fig. 1, when displaying quantized multilevel elements the available phase domain may be smaller than $360^{\circ}$, i.e., this constraint may be relaxed if necessary. In Fig. 2 we show the phase depth required as a function of the number of quantization levels of the multilevel diffractive element. The lower limit corresponds to the case of the binary phase element, where a $180^{\circ}$ phase depth domain is required. For 6 and 12 quantization levels, the phase depth necessary is, respectively, $300^{\circ}$ and $330^{\circ}$. We note that first diffraction efficiency for blazed gratings with 6 and 12 quantization levels reaches such high values as $\eta_{1}=91 \%$ and $\eta_{1}=98 \%$, respectively.

To perform the experiments in the lab, a light beam from a He-Ne laser at $633 \mathrm{~nm}$ is expanded using a spatial filter and then is collimated with a lens. As given in Section 2 , the angle of incidence onto the LCoS is $3^{\circ}$. The collimated beam of light passes through a polarizer whose transmission axis is oriented so that the incident SOP corresponds to linearly polarized light along the lab-horizontal, i.e., parallel to the director axis of the liquid crystal in the LCoS: this is the phase-only configuration. We have checked that there is no need for an analyzer

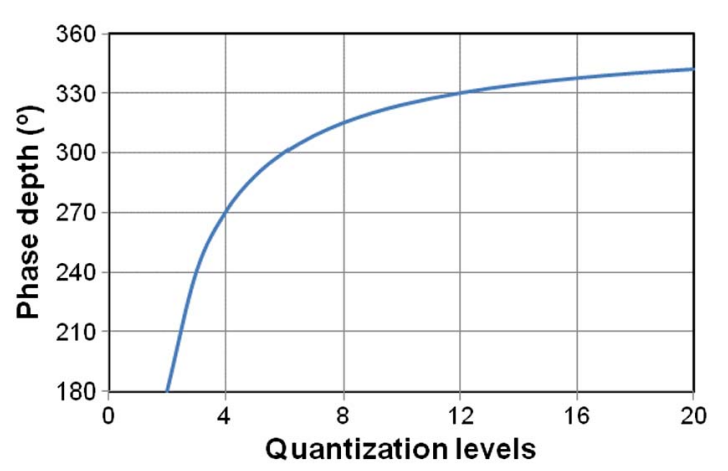

Fig. 2. Phase depth domain necessary as a function of the number of quantization levels of the multilevel phase element. 
at the output since the reflected SOP is fully linearly polarized along the lab-horizontal. A lens focuses the reflected beam to its focal plane where a detector connected to a radiometer measures the diffracted intensity.

We have measured both zero- and first-order intensities in the following ( $I_{0}$ and $I_{1}$, respectively) for a series of blazed gratings with a spatial frequency of $2,3,4,8,12$, and 16 pixels/period, thus covering both a wide spatial frequency and number of quantization levels range. A diaphragm in front of the detector is needed to filter out any unwanted orders in the measurements. Vertical blazed gratings are addressed, i.e., the spatial frequency corresponds to rows/period, since along this direction potential deviations due to the anamorphic frequency-dependent effect [26-28] are less important. In this paper we want to isolate as much as possible the flicker from other degradation effects which may be exhibited by SLMs. In this sense the anamorphic frequencydependent effect has been found both in transmissive LCDs [26] and in modern LCoS devices, both analog [27] and digitally [28] driven.

The values obtained, $I_{0}$ and $I_{1}$, need to be normalized by the total energy reflected by the LCoS. To this goal we apply a uniform zero gray-level value screen, then all the light reflected by the LCoS is sent to the zero order. We call this intensity value $I_{T 1}$. We note that due to the pixelated structure of the device, multiple replicas of the diffraction orders of the blazed grating are generated, each replica centered at the position of one of the diffraction orders produced by the periodic pixelated structure. This is a well-known consequence of the sampling theory [29]. We restrict our attention to the zero-order replica, i.e., the one centered at the position of the zero order produced by the periodic pixelated structure. In principle the relative energy deflected to each of the replicas does not depend on the image being addressed but only on geometrical characteristics of the display, basically on the fill factor. However, during the realization of the experiment in the lab we observed a very clear change of brightness between different replicas when a uniform screen was sent with respect to sending the blazed gratings. Therefore we measured the total energy in the zero-order replica in the following $I_{T 2}$ when addressing a blazed grating: we open the aperture of the diaphragm in front of the detector so that only the set of diffraction orders in the zero-order replica are gathered. Specifically, we have measured $I_{T 2}$ for the lowest spatial frequency blazed grating, 16 pixels/period, since aliasing between neighboring replicas is then reduced; thus the $I_{T 2}$ value is more robust. In principle, according to the sampling theory the values $I_{T 1}$ and $I_{T 2}$ should be the same; however we found their ratio to be $\mathrm{R}=I_{T 2} / I_{T 1}=0.92$, i.e., almost a $10 \%$ of the energy was deflected into higher-order replicas. Therefore, additional care has to be taken when normalizing the measurements to compensate for this unexpected exchange of energy between replicas. Then, the normalized intensity (or diffraction efficiency) can be obtained as

$$
\eta_{i}=\frac{1}{\mathrm{R}} \frac{I_{i}}{I_{T 1}}
$$

where $i$ is the number of the diffraction order considered for the blazed grating. Probably the different exchange of energy between replicas when changing the image being addressed onto the LCoS is due to the pixel crosstalk caused by the fringing fields, i.e., the gradual voltage changes across the border of neighboring pixels and by elastic forces in the liquid-crystal material preventing abrupt spatial variations in the phase modulation [30-33]. Both effects cause the realized phase modulation of a pixel to depend also on the voltage applied over adjacent pixels. This crosstalk phenomenon increases as pixels get smaller and as voltage changes between neighboring pixels are larger.

In Figs. 3(a) and 3(b) we show respectively for the sequences "18-6 633 2pi linear" and "5-5 633 2pi linear" the results for the measurements of the instantaneous first-order diffraction efficiencies for the six blazed gratings. In the legend we show the quantization number of levels, $q$, in each case. All the gratings are measured and optimized for the wavelength $633 \mathrm{~nm}$. The instantaneous values have been obtained by connecting a radiometer model 1830-C from Newport, that has an analog bandwidth greater
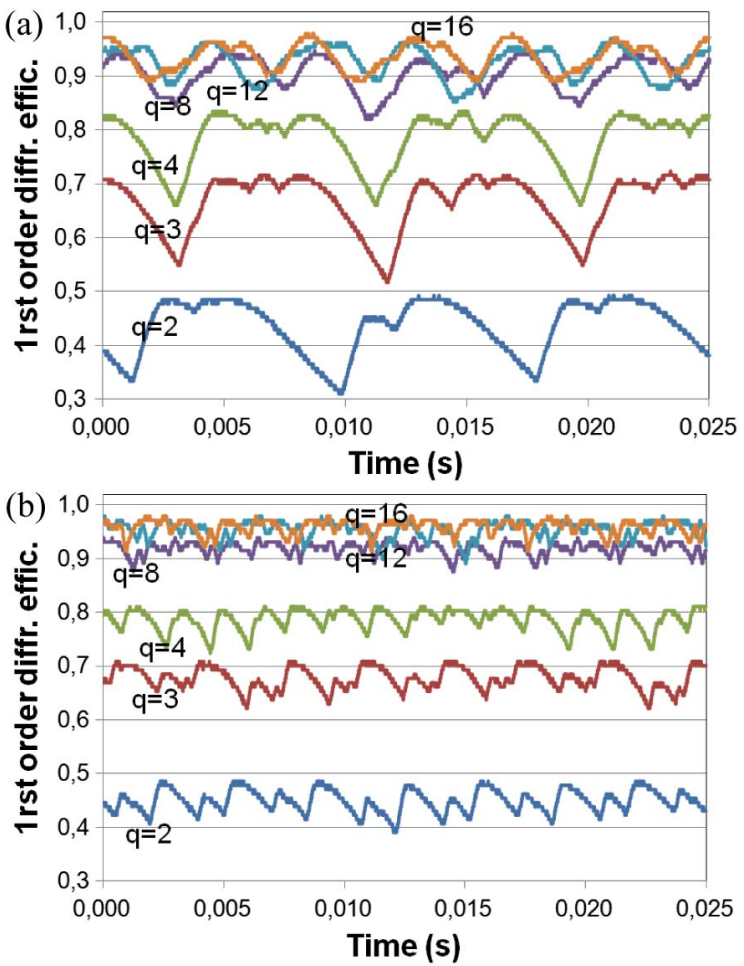

Fig. 3. Instantaneous values for the first-order diffracted intensity for blazed gratings with different quantization levels ( $q$ shown in the legend); (a) sequence "18-6 633 2pi linear;" (b) sequence "5-5 6332 pi linear." Data for $\lambda=633 \mathrm{~nm}$ and for quasi-perpendicular incidence at $3^{\circ}$. 
than $30 \mathrm{kHz}$, to a digital oscilloscope model TDS$1012 \mathrm{~b}$ from Tektronix, with a $100 \mathrm{MHz}$ analog bandwidth. The first thing we note is that the diffracted intensity fluctuates, with a period of $8.33 \mathrm{~ms}$, i.e., frequency $120 \mathrm{~Hz}$. The amplitude of fluctuations in Figs. 3(a) and 3(b) is smaller as the number of quantization levels increases. Additionally, the average diffraction also increases with $q$ which is the theoretically expected result. If we compare the plots for the two sequences, we find that the amplitude in the oscillations is smaller for the 5-5 configuration file.

Curves in Figs. 3(a) and 3(b) have been acquired with arbitrary triggers and they do not have the same starting instant. This is not relevant for the analysis in the present paper where the various quantized gratings are not to be addressed simultaneously. However it is interesting to note that there are applications where proper synchronization of acquisition times in the analysis may be an issue as given by [34], which shows empirically that for polychromatic illumination applications, fluctuations given by a diffraction grating for the various wavelengths may be partly synchronized by addressing the diffraction grating with the appropriate gray levels for each separate wavelength.

For the simulations, first we calculate the gray level $g$ to be applied to produce the desired phase value $\varphi$ at each step in the multilevel blazed grating. To this goal we consider the calibrated values $\bar{\Gamma}(g)$ given in Fig. 1, and for each desired $\varphi$ we look for the closest available average retardance value $\bar{\Gamma}$. Second, the frame period $T$ is divided in time intervals small enough so that the fluctuating phase profile of the blazed grating is accurately sampled. At each time sample, the staircase phase profile is partly distorted from the ideal quantized linear ramp since each step, associated with a specific gray level $g$, is fluctuating with a different amplitude $a(g)$, given in Fig. 1 . The various phase steps in the staircase profile fluctuate in a correlated form with each other, in accordance with the periodic behavior exhibited by the instantaneous values in Fig. 3 . As a starting value, at time $t=0$ we have chosen for each phase step $\varphi$ the phase value $\bar{\Gamma}(g)-a(g)$ which then increases linearly with time until $\bar{\Gamma}(g)+a(g)$. Another starting set of values may be chosen, resulting in different instantaneous profiles, but since we are interested in averaged effects this has no relevance on the results. As a third and final part of the simulation, at each time sample the Fourier transform of the blazed grating profile is calculated: this provides the instantaneous diffraction efficiency. From these instantaneous values we calculate the averaged one and the amplitude and standard deviation for its fluctuation. Quantitative comparisons between simulations and experiment are presented in the next section.

\section{Results and Discussion}

We want to perform a complete analysis of the suitability of the calibration performed using the average
Stokes polarimetric technique and also to obtain a deeper insight on the actual degree of influence of fluctuations on the performance of blazed gratings, as a widespread example of a multilevel phase element. To fulfill these two goals we look both into average diffraction efficiencies and also the magnitude of its variations, i.e., we plan both static and dynamic analyses. Depending on the application it is not only important to maximize first-order diffraction efficiency and/or to reduce the importance of the zero order, but it may be also important to generate stable spots. One such application is holographic optical tweezers where both intensity and positional stability is an issue [8]. In the case of an optical communication link, where the LCoS is playing the role of a reconfigurable optical add-drop multiplexer (ROADM) [5], these fluctuations lead to a degradation of the signal-to-noise ratio and a nondesirable increase in the bit-error rate (BER) in the communication link.

Let us start with the results dealing with the static analysis, i.e., average diffraction efficiencies. In Figs. 4(a) and 4(b) we show the diffraction efficiency for the zero order and for the first order, respectively as a function of the quantization levels of the blazed grating and for the two configuration files addressed. Lines and markers correspond, respectively, to simulated and experimental results. The simulated results take into account both the average retardance and the flicker amplitude values shown in Fig. 1 . The experimental results correspond to the average value delivered on the display of the radiometer. In
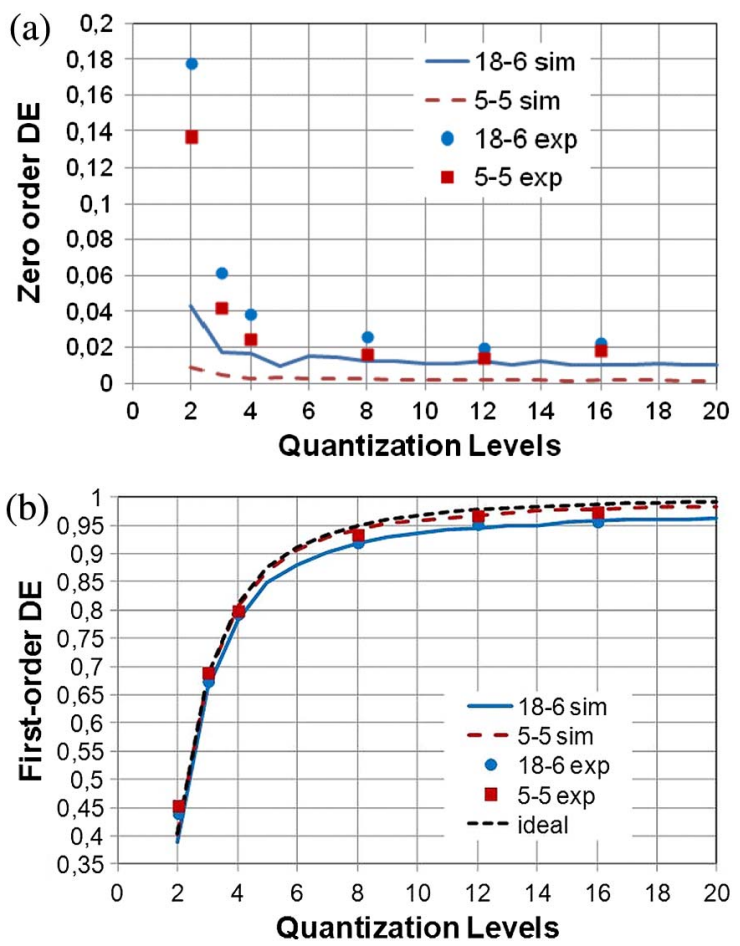

Fig. 4. Realistic simulations and experimental results for the diffraction efficiency for the two configuration files; (a) zero order; (b) first order. 
relation with Fig. 4(a) let us remember that for a $2 \pi$ phase depth blazed grating the theoretical diffraction efficiency for the zero order is $\eta_{0}=0$. The theoretical simulation taking into account the existence of flicker provides values that are nonzero, being slightly more intense for the 18-6 sequence. We note that the simulated values decrease with the number of quantization values. The same tendency can be appreciated in the experimental results, which show values equal or smaller than $2 \%$ for eight quantization levels and above, which is a small residual zero order. For less than five quantization levels the deviation between simulation and experiment increases. This may be partly due to the aliasing effect between diffraction orders from neighboring pixelation replicas.

In Fig. 4(b) we show the equivalent plot corresponding to the first-order diffraction efficiency. We see that the experimental values show a very good agreement with simulations, especially for quantization levels equal or larger than 4 . We also note in the experimental values that the efficiency is slightly larger for the 5-5 sequence, which agrees with the simulation results. The theoretical curve given by Eq. (4) is also included, "ideal" label in the legend, to show the actual effect of fluctuations on the diffraction efficiency. We see that for the sequence $5-5$, fluctuations have basically no effect in diminishing the diffraction efficiency. To make this important result more evident, in Fig. 5 we represent the deviation between the ideal diffraction efficiency and the realistic numerical simulation, which is numerically calculated for each of the two sequences including the calibrated fluctuations, normalized by the ideal diffraction efficiency. We see that the normalized difference for the sequence 18-6 decreases from $4.5 \%$ to $3 \%$ as the number of quantization levels increase. In the case of the sequence 5-5, the normalized difference shows a constant behavior and the values are smaller than $1 \%$. Thus, it can be concluded that for the latter sequence fluctuations are not actually degrading the performance of the element. If the amplitude of fluctuations is larger, as it is the case for the sequence 18-6, it is important to note

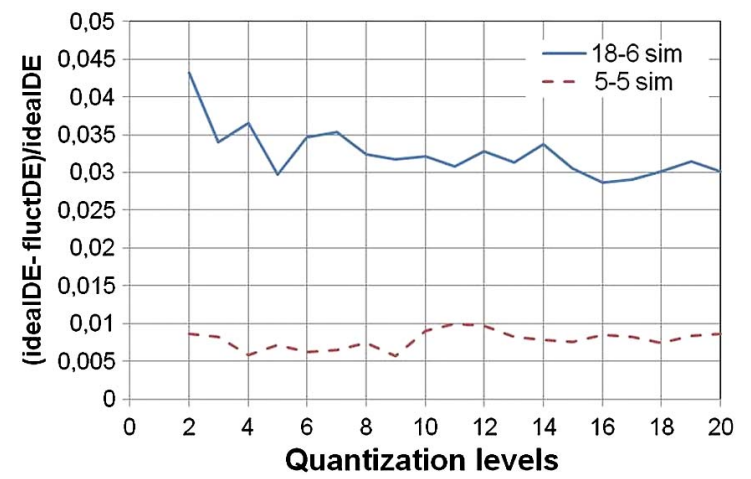

Fig. 5. For each of the two sequences, deviation between the ideal first-order diffraction efficiency and the realistic numerical simulation (i.e., including the calibrated fluctuations), normalized by the ideal diffraction efficiency. that their effect decreases as the number of quantization levels increase, approaching reasonable values for five quantization levels or larger.

A possible criticism to the previous analysis regarding deviations between ideal values [given by Eq. (4)] and the realistic simulated values is that the latter are not only affected by fluctuations, but also by the mismatch between the required and the available phase value for each level. This mismatch is induced by the limited and nonequally spaced quantization levels in the available phase domain. Let us note that the number of phase levels is limited to 256 for configuration 18-6 and 92 for configuration 5-5 as commented in Section 2, and they do not need to be equally spaced in the phase domain. The small plateaus for the average retardance in Fig. 1 show this limited and nonequal spacing in the available phase domain. In Fig. $\underline{6}$ we show for the two sequences, the numerically calculated difference between the ideal diffraction efficiency and the one actually given by the available phase domain, normalized by the ideal diffraction efficiency. In these simulations we consider that there are no fluctuations and only the calibrated average retardance values are used, thus the only deviations result from the mismatch between required and available phase values for each of the phase levels. We see that the normalized deviation is smaller than $0.1 \%$, being slightly larger for the sequence 5-5, which is the one with a smaller number of phase levels. This means that the phase levels mismatch effect can be disregarded when discussing the influence of fluctuations on the diffraction efficiency.

In the first paragraph in this section we commented about the necessity for a "static" and a "dynamic" analysis of the influence of flicker on the performance of the elements addressed. Previous plots dealing with average diffraction efficiencies focus on the "static" analysis. Next we show in Figs. 7(a) and 7(b), for the first diffraction order of the displayed blazed gratings, respectively, the amplitude of the fluctuations and the standard deviation values, normalized by the average diffraction efficiency. Experimentally, the average and the fluctuating estimation parameters are obtained from

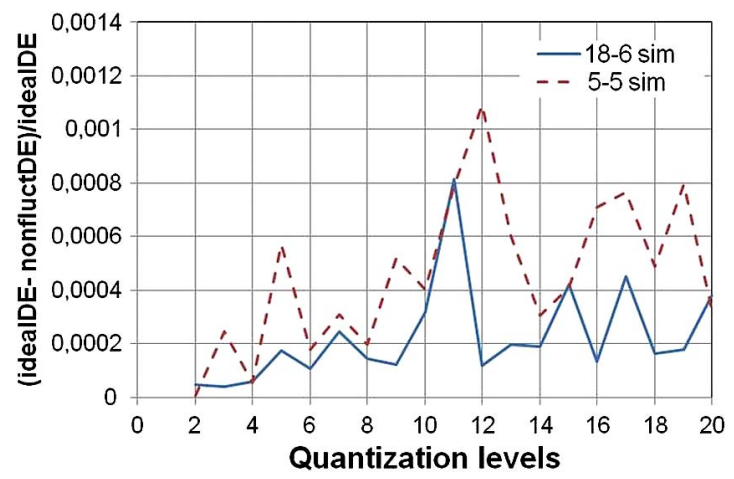

Fig. 6. For each of the two sequences, deviation between the ideal first-order diffraction efficiency and the numerical simulation (including only the calibrated average retardance), normalized by the ideal diffraction efficiency. 

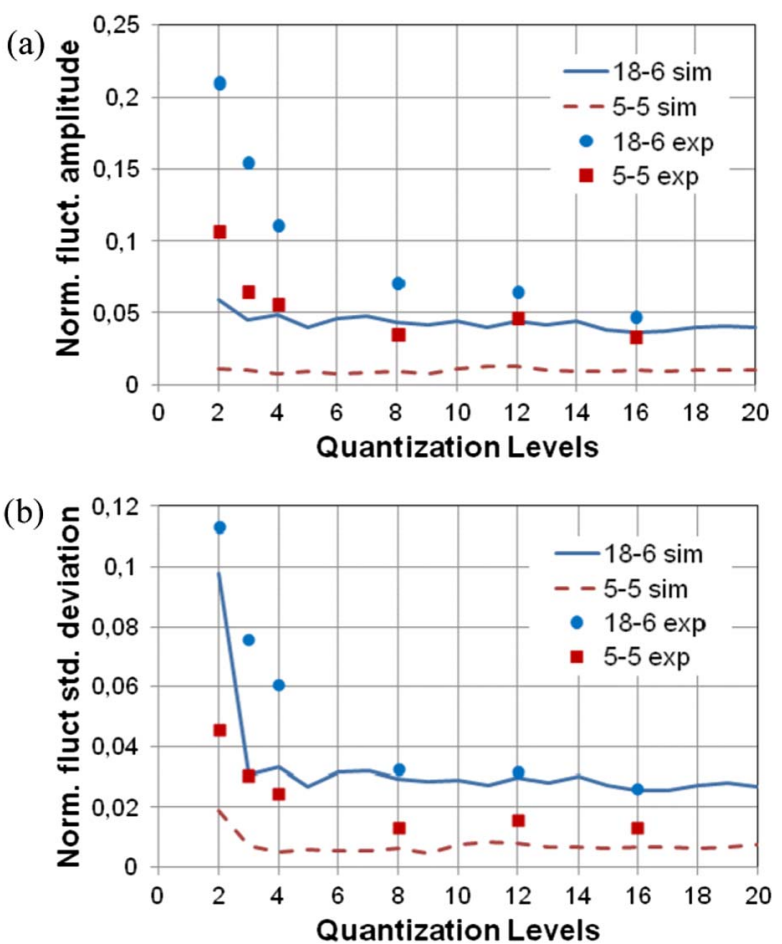

Fig. 7. For the two configuration files, realistic simulations and experimental results for the magnitude of the time fluctuation in the first-order diffraction efficiency normalized by its average value; (a) amplitude; (b) standard deviation.

the measurements acquired with the digital oscilloscope, shown in Fig. 3. Numerically, as commented at the end of Section $\overline{3}$, the instantaneous values are simulated applying the triangular time evolution of the fluctuating retardance using (for each gray level) the average retardance $\bar{\Gamma}$ and its fluctuation amplitude $a$ (characterized in Fig. 1). From these diffraction efficiency instantaneous values we calculate the average value, the amplitude, and the standard deviation for its fluctuation. The amplitude and standard deviation are then normalized by the average value.

In Fig. 7(a) simulation and experiment show a good agreement when using more than eight quantization levels. Both numerically and experimentally we obtain that sequence 5-5 shows smaller fluctuations. In Fig. 7(b), where the figure-of-merit is the standard deviation, the agreement between experimental and numerical results is very good, especially for more than eight quantization levels. The tendency is clear: to reduce fluctuations generated by the optical element addressed the number of quantization levels has to be larger than 5. In this case the time stability in the spot reaches a low value for the sequence 18-6 and becomes very good for the sequence 5-5. We can conclude that standard deviation is a much more robust figure-of-merit since it takes a more averaged estimation of the time fluctuations. It is important to remark that the results in Fig. 7 are not dependent on the external normalization factor $R$ shown in Eq. (), which is due to the crosstalk effect between neighboring pixelation replicas. This gives even more relevance to the excellent agreement between simulation and experiment in Fig. 7(b) since they are self-referenced measurements without an additional parameter which may introduce further uncertainty in the results.

\section{Conclusions}

We have shown that the average retardance and its fluctuation value calibrated by means of the average Stokes polarimetry technique can be used to predict the diffraction efficiency for multilevel phase blazed gratings, not only its average ("static" analysis) but also its time fluctuation ("dynamic" analysis). This is very important since it enables calculating in advance if the specific LCoS device and/or the configuration of the electrical parameters governing its performance may be the appropriate ones for a specific application. An interesting result obtained is that the degradation produced by a sequence possessing a large amount of flicker can be largely decreased, even to acceptable values, if the number of quantization levels in the element is increased, as we have seen for the sequence 18-6: for five or more quantization levels the decrease both in the first-order diffraction efficiency and in the magnitude of time fluctuations was kept small. Then, for the sequence 5-5, whose retardance flicker amplitude is lower than $30^{\circ}$, we have obtained that the diffraction efficiency results are very close to the ones that may be expected in the ideal case with no fluctuations. As a byproduct of the present work we have seen that care has to be taken in the experiments due to the crosstalk between neighboring pixelation replicas. Proper normalization enabled us to uncouple this crosstalk from the analysis of flicker influence in spatially varying phase elements.

This work was supported by the Ministerio de Trabajo y Competitividad of Spain (Projects FIS2011-29803-C02-01 and FIS2011-29803-C02-02), by the Generalitat Valenciana of Spain (Projects PROMETEO/2011/021 and ISIC/2012/013), and by the University de Alicante (Project GRE12-14).

\section{References}

1. J. Turunen and F. Wyrowski, eds., Diffractive Optics for Industrial and Commercial Applications (Akademie, 1997).

2. J. Joseph and D. A. Waldman, "Homogenized Fourier transform holographic data storage using phase spatial light modulators and methods for recovery of data from the phase image," Appl. Opt. 45, 6374-6380 (2006).

3. K. Curtis, L. Dhar, A. Hill, W. Wilson, and M. Ayres, eds., Holographic Data Storage: From Theory to Practical Systems (Wiley, 2010).

4. W. Osten, C. Kohler, and J. Liesener, "Evaluation and application of spatial light modulators for optical metrology," Opt. Pura Appl. 38, 71-81 (2005).

5. M. A. F. Roelens, S. Frisken, J. A. Bolger, D. Abakoumov, G. Baxter, S. Poole, and B. J. Eggleton, "Dispersion trimming in a reconfigurable wavelength selective switch,” J. Lightwave Technol. 26, 73-78 (2008).

6. M. Salsi, C. Koebele, D. Sperti, P. Tran, H. Mardoyan, P. Brindel, S. Bigo, A. Boutin, F. Verluise, P. Sillard, M. BigotAstruc, L. Provost, and G. Charlet, "Mode-division multiplexing 
of $2100 \mathrm{~Gb} / \mathrm{s}$ channels using an LCOS-based spatial modulator," J. Lightwave Technol. 30, 618-623 (2012).

7. A. Dudley, G. Milione, R. R. Alfano, and A. Forbes, "All-digital wavefront sensing for structured light beams," Opt. Express 22, 14031-14040 (2014)

8. A. Farré, M. Shayegan, C. López-Quesada, G. A. Blab, M. Montes-Usategui, N. R. Forde, and E. Martín-Badosa, "Positional stability of holographic optical traps," Opt. Express 19, 21370-21384 (2011).

9. M. A. Solís-Prosser, A. Arias, J. J. M. Varga, L. Rebón, S. Ledesma, C. Iemmi, and L. Neves, "Preparing arbitrary pure states of spatial qudits with a single phase-only spatial light modulator," Opt. Lett. 38, 4762-4765 (2013).

10. N. Collings, T. Davey, J. Christmas, D. Chu, and B. Crossland, "The applications and technology of phase-only liquid crystal on silicon devices,” J. Disp. Technol. 7, 112-119 (2011).

11. J. E. Wolfe and R. A. Chipman, "Polarimetric characterization of liquid-crystal-on-silicon panels," Appl. Opt. 45, 1688-1703 (2006).

12. A. Hermerschmidt, S. Osten, S. Krüger, and T. Blümel, "Wave front generation using a phase-only modulating liquid-crystal based micro-display with HDTV resolution," Proc. SPIE 6584, $65840 \mathrm{E}(2007)$

13. J. R. Moore, N. Collings, W. A. Crossland, A. B. Davey, M. Evans, A. M. Jeziorska, M. Komarčević, R. J. Parker, T. D. Wilkinson, and $\mathrm{H}$. Xu, "The silicon backplane design for an LCOS polarization-insensitive phase hologram SLM," IEEE Photon. Technol. Lett. 20, 60-62 (2008).

14. A. Márquez, I. Moreno, C. Iemmi, A. Lizana, J. Campos, and M. J. Yzuel, "Mueller-Stokes characterization and optimization of a liquid crystal on silicon display showing depolarization," Opt. Express 16, 1669-1685 (2008).

15. P. Clemente, V. Durán, Ll. Martínez-León, V. Climent, E. Tajahuerce, and J. Lancis, "Use of polar decomposition of Mueller matrices for optimizing the phase response of a liquidcrystal-on-silicon display," Opt. Express 16, 1965-1974 (2008).

16. A. Lizana, I. Moreno, A. Márquez, C. Iemmi, E. Fernández, J. Campos, and M. J. Yzuel, "Time fluctuations of the phase modulation in a liquid crystal on silicon display: characterization and effects in diffractive optics," Opt. Express 16, 1671116722 (2008)

17. A. Lizana, I. Moreno, A. Márquez, E. Also, C. Iemmi, J. Campos, and M. J. Yzuel, "Influence of the temporal fluctuations phenomena on the ECB LCoS performance," Proc. SPIE 7442 $74420 \mathrm{G}(2009)$.

18. J. García-Márquez, V. López, A. González-Vega, and E. Noé, "Flicker minimization in an LCoS spatial light modulator," Opt. Express 20, 8431-8441 (2012).

19. F. J. Martínez, A. Márquez, S. Gallego, J. Francés, and I. Pascual, "Extended linear polarimeter to measure retardance and flicker: application to liquid crystal on silicon devices in two working geometries," Opt. Eng. 53, 014105 (2014).

20. C. Ramirez, B. Karakus, A. Lizana, and J. Campos, "Polarimetric method for liquid crystal displays characterization in presence of phase fluctuations," Opt. Express 21, 31823192 (2013).

21. F. J. Martínez, A. Márquez, S. Gallego, J. Francés, I. Pascual, and A. Beléndez, "Retardance and flicker modeling and characterization of electro-optic linear retarders by averaged Stokes polarimetry," Opt. Lett. 39, 1011-1014 (2014).

22. F. J. Martínez, A. Márquez, S. Gallego, M. Ortuño, J. Francés, A. Beléndez, and I. Pascual, "Averaged Stokes polarimetry applied to evaluate retardance and flicker in PA-LCoS devices," Opt. Express 22, 15064-15074 (2014).

23. A. Lizana, A. Márquez, L. Lobato, Y. Rodange, I. Moreno, C. Iemmi, and J. Campos, "The minimum Euclidean distance principle applied to improve the modulation diffraction efficiency in digitally controlled spatial light modulators," Opt. Express 18, 10581-10593 (2010).

24. G. Goldstein, Polarized Light (Marcel Dekker, 2003).

25. F. J. Martínez, A. Márquez, S. Gallego, M. Ortuño, J. Francés, A. Beléndez, and I. Pascual, "Electrical dependencies of optical modulation capabilities in digitally addressed parallel aligned LCoS devices," Opt. Eng. 53, 067104 (2014).

26. A. Márquez, C. Iemmi, I. Moreno, J. Campos, and M. J. Yzuel, "Anamorphic and spatial frequency dependent phase modulation on liquid crystal displays. Optimization of the modulation diffraction efficiency," Opt. Express 13, 21112120 (2005).

27. J. Albero, P. García-Martínez, J. L. Martínez, and I. Moreno, "Second order diffractive optical elements in a spatial light modulator with large phase dynamic range," Opt. Lasers Eng. 51, 111-115 (2013).

28. L. Lobato, A. Lizana, A. Márquez, I. Moreno, C. Iemmi, J. Campos, and M. J. Yzuel, "Characterization of the anamorphic and spatial frequency dependent phenomenon in liquid crystal on silicon displays," J. Eur. Opt. Soc. Rapid Pub. 6, 11012S (2011).

29. J. W. Goodmann, Introduction to Fourier-Optics, 3rd ed. (Roberts, 2005).

30. B. Apter, U. Efron, and E. Bahat-Treidel, "On the fringingfield effect in liquid-crystal beam-steering devices," Appl. Opt. 43, 11-19 (2004).

31. U. Efron, B. Apter, and E. Bahat-Treidel, "Fringing-field effect in liquid-crystal beam-steering devices: an approximate analytical model," J. Opt. Soc. Am. A 21, 1996-2008 (2004).

32. M. Persson, D. Engström, and M. Goksör, "Reducing the effect of pixel crosstalk in phase only spatial light modulators," Opt. Express 20, 22334-22343 (2012).

33. C. Lingel, T. Haist, and W. Osten, "Optimizing the diffraction efficiency of SLM-based holography with respect to the fringing field effect," Appl. Opt. 52, 6877-6883 (2013).

34. K. Kakarenko, M. Zaremba, I. Ducin, M. Makowski, A. Siemion, A. Siemion, J. Suszek, M. Sypek, D. Wojnowski, Z. Jaroszewicz, and A. Kolodziejczyk, "Utilization of the phase flicker of a LCoS spatial light modulator for improved diffractive efficiency," Photon. Lett. Pol. 2, 128-130 (2010). 